\title{
A comparative study on use of two versus three double-loaded suture anchors in arthroscopic Bankart repair
}

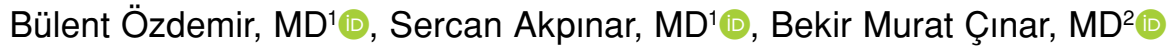 \\ 1'Department of Orthopedics and Traumatology, Medline Hospital, Adana, Turkey \\ ${ }^{2}$ Department of Orthopedics and Traumatology, Başkent University Adana Hospital, Adana, Turkey
}

Traumatic anterior instability or dislocation of the shoulder joint usually results in avulsion of the anterior inferior labrum. Bankart defined this lesion as the essential lesion of shoulder instability. ${ }^{[1]}$ Recently, arthroscopic Bankart repair has been frequently performed for anterior shoulder instabilities, and the results similar to those for open surgical repair have been reported. ${ }^{[2]}$ Different types of suture anchors are used in arthroscopic Bankart repair. Currently, metallic, bioabsorbable, double-loaded or single-loaded, and knotless suture anchors are available. There is no standardization for the number of suture anchors to be used in arthroscopic Bankart repair. The number and type of suture anchors used depend on the surgeon's knowledge and experience. Although there is no definite number of suture anchors required in arthroscopic Bankart repair, a minimum of three suture anchors have been recommended in many publications..$^{[3-5]}$ Although the use of three or

Received: April 18, 2020

Accepted: October 27, 2020

Published online: January 06, 2021

Correspondence: Bülent Özdemir, MD. Güzelyalı Mah., 81190 Sok., Safir Deluxe Sitesi, A Blok, Daire: 34, 01170 Çukurova, Adana.

E-mail: bulent0116@hotmail.com

Doi: $10.5606 /$ ehc. 2021.75536

Citation: Özdemir B, Akpınar S, Çınar BM. A comparative study on use of two versus three double-loaded suture anchors in arthroscopic Bankart repair. Jt Dis Relat Surg 2021;32(1):108-114.

(02021 All right reserved by the Turkish Joint Diseases Foundation

This is an open access article under the terms of the Creative Commons Attribution-NonCommercial License, which permits use, distribution and reproduction in any medium, provided the original work is properly cited and is not used for commercial purposes (http://creativecommons.org/licenses/by-nc/4.0/).

\section{ABSTRACT}

Objectives: This study aims to compare clinical results of repair using two versus three double-loaded suture anchors in arthroscopic Bankart repair.

Patients and methods: Between July 2012 and December 2017, a total of 40 patients (38 males, 2 females; mean age: $31.6 \pm 8.1$; range: 17 to 47 years) who underwent Bankart arthroscopic surgery and were followed for minimum two years were retrospectively analyzed. Group $1(n=17)$ underwent arthroscopic Bankart repair with two double-loaded suture anchors, while Group $2(n=23)$ underwent repair with three double-loaded suture anchors. Clinical outcomes of the patients and recurrences were compared.

Results: At the final postoperative follow-up, a significant improvement was observed in the functional outcomes in all patients. No statistically significant difference was found $(\mathrm{p}>0.05)$ in the mean clinical scores of the Constant Shoulder Score between Group $1(94.2 \pm 7.8)$ and Group $2(95.4 \pm 4.1)$. There was no significant difference in the mean Rowe scores (Group 1: 95.6 \pm 4.6 vs. Group 2: $96.3 \pm 3.8$, respectively) and external rotation loss (at neutral Group 1: $1.9^{\circ}$ vs. Group 2: $2.2^{\circ}$, respectively). Three of our patients had recurrent dislocation during a major traumatic event ( $\mathrm{n}=2$ in Group 1 and $\mathrm{n}=1$ in Group 2).

Conclusion: Our study results suggest that stability is not correlated with the use of either two versus three double-loaded suture anchors in arthroscopic Bankart repairs.

Keywords: Anterior shoulder dislocation, Bankart repair, chronic shoulder instability, double-loaded anchor, shoulder arthroscopy.

more suture anchors is recommended as the gold standard, there are also studies showing that similar clinical results are obtained with surgeries performed with one or two suture anchors. However, some studies have shown that the use of one or two suture anchors provides sufficient stability and successful clinical outcomes have been reported..$^{[6,7]}$ Aydin et al. ${ }^{[8]}$ compared the use of knotless two and three suture anchors in arthroscopic Bankart repair. In this study, although similar clinical results were observed 
between the groups, they found a higher number of recurrence in cases where two suture anchors were used. Arthroscopic knot-tying techniques and configurations have been extensively studied. ${ }^{[9]}$ Nho et al. ${ }^{\left[{ }^{[9]}\right.}$ studied the biomechanical properties of different knot shapes, and found no statistically significant difference. However, they observed a significant biomechanical difference between double-loaded and single-loaded suture anchors in their study.

In the present study, we hypothesized that the use of two suture anchors would not make a difference in clinical outcomes compared to three suture anchors. We, therefore, aimed to compare the clinical results between two double-loaded suture anchors and three double-loaded suture anchors in arthroscopic Bankart repair.

\section{PATIENTS AND METHODS}

This single-center, retrospective study was conducted at Adana Medline Hospital between July 2012 and December 2017. A total of 53 patients who underwent arthroscopic Bankart repair due to traumatic recurrent anterior shoulder dislocation were retrospectively analyzed. Patients who completed at least 24-month follow-up were included in the study. All patients had anteroinferior labral Bankart tears in similar sizes between the 3 and 6 o'clock position of the glenoid. Those with large bone defects, multidirectional shoulder instability, capsular laxity, large Hill-Sacs lesions (larger than $5 \mathrm{~mm}$ ), rheumatologic disease, rotator cuff tears, glenohumeral arthrosis, glenoid rim fracture, neurological deficit, superior labral anterior posterior lesions, and recurrent instability after Bankart repair were excluded. Finally, a total of 40 patients (38 males, 2 females; mean age: $31.6 \pm 8.1$; range: 17 to 47 years) who underwent Bankart arthroscopic surgery and were followed for minimum two years were included in the study. A written informed consent was obtained from each patient. The study protocol was approved by the Adana Medline Hospital Ethics Committee (2020/996). The study was conducted in accordance with the principles of the Declaration of Helsinki.

The patients were divided into two groups according to the number of suture anchors used. Group $1 \quad(n=17)$ underwent arthroscopic Bankart repair with two double-loaded suture anchors, while Group $2(n=23)$ underwent arthroscopic Bankart repair with three double-loaded suture anchors.

The patients were evaluated postoperatively using the Constant Shoulder Score (CSS) and
Rowe Score (RS). In all patients, the range of motion (ROM) of the operated shoulders was evaluated. The postoperative evaluation of the patients was performed by the same surgeons. The clinical outcomes were evaluated in each group.

In all patients, preoperative radiographs of the shoulder were obtained to detect if any bony lesions or arthritis were present. All instabilities found, in any of the patients, were routinely evaluated by magnetic resonance imaging before surgery to identify labral Bankart lesions and diagnose concomitant abnormalities.

\section{Surgical technique}

All operations were performed in the beach chair position under general anesthesia. The standard arthroscopic portals including posterior, anterosuperior, and anteroinferior portals were established. Diagnostic arthroscopy was performed to assess intraarticular pathology. The capsulolabral tissue was mobilized, from the anterior glenoid surface, with an electrocautery and an arthroscopic periosteal elevator. The anterior glenoid bone was decorticated using a motorized shaver or a $4.5 \mathrm{~mm}$ round burr. In all cases, the capsulolabral tissue was repaired using suture anchors by Samsung Medical Center (SMC; Samsung Medical Center, Seoul, Korea) knots.

Anchor holes were created on the anterior glenoid edge using a drill of an angle of 50 to 70 degrees to the plane of the glenoid. Labrum was penetrated to the most inferior position with a $45^{\circ}$ curved suture passing device (ACCU-PASS ${ }^{\mathrm{TM}}$; Smith \& Nephew, Memphis, TN, USA) through the anteroinferior portal. A No.2 non-absorbable suture (ULTRABRAID $^{\text {TM}}$; Smith \& Nephew, Memphis, TN, USA) was, then, passed through the capsulolabral complex by passing from the anteroinferior portal. Both ends of the suture anchors were tied through the anteroinferior portal using the SMC knot. The other anchors were inserted appropriately in sequence from inferior to superior. In Group 1, two double-loaded suture anchors were used, while three double-loaded suture anchors were used in Group 2 (Figures 1 and 2).

The tensioning of the anterior capsulolabral complex was established by pulling the capsulolabral tissue with a grasper in the direction of the anterosuperior portal. Then a careful examination, using a probe, was made in all patients to ensure that the repair was successful, capsulolabral complex was stable, and the labral hump was restored. Routine closure of the portals was performed. 
(a)

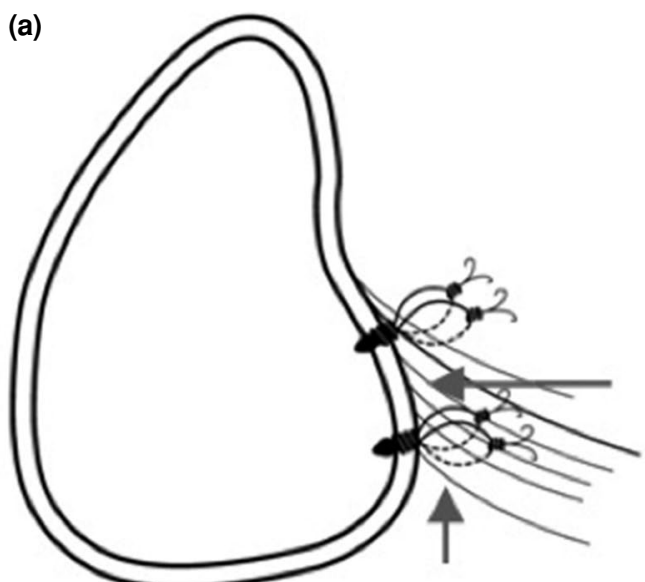

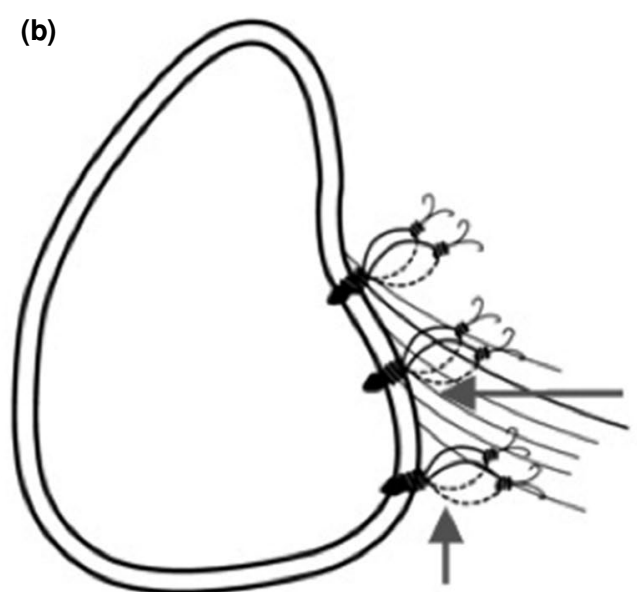

(b)

FIGURE 1. In the study, both two and three double-loaded suture anchors were used, providing both medial and superior shift of the capsule. (a) Group 1 underwent an arthroscopic Bankart repair with two double-loaded suture anchors. (b) Group 2 underwent an arthroscopic Bankart repair with three double-loaded suture anchors.

\section{Postoperative rehabilitation}

All patients were discharged one day after the operation. Shoulders of all patients were immobilized with a simple arm sling for four weeks. The
ROM-enhancing and muscle-strengthening exercises were started within four weeks. We attempted to reach the full shoulder $\mathrm{ROM}$ at eight weeks with passive exercises and, particularly, we achieved
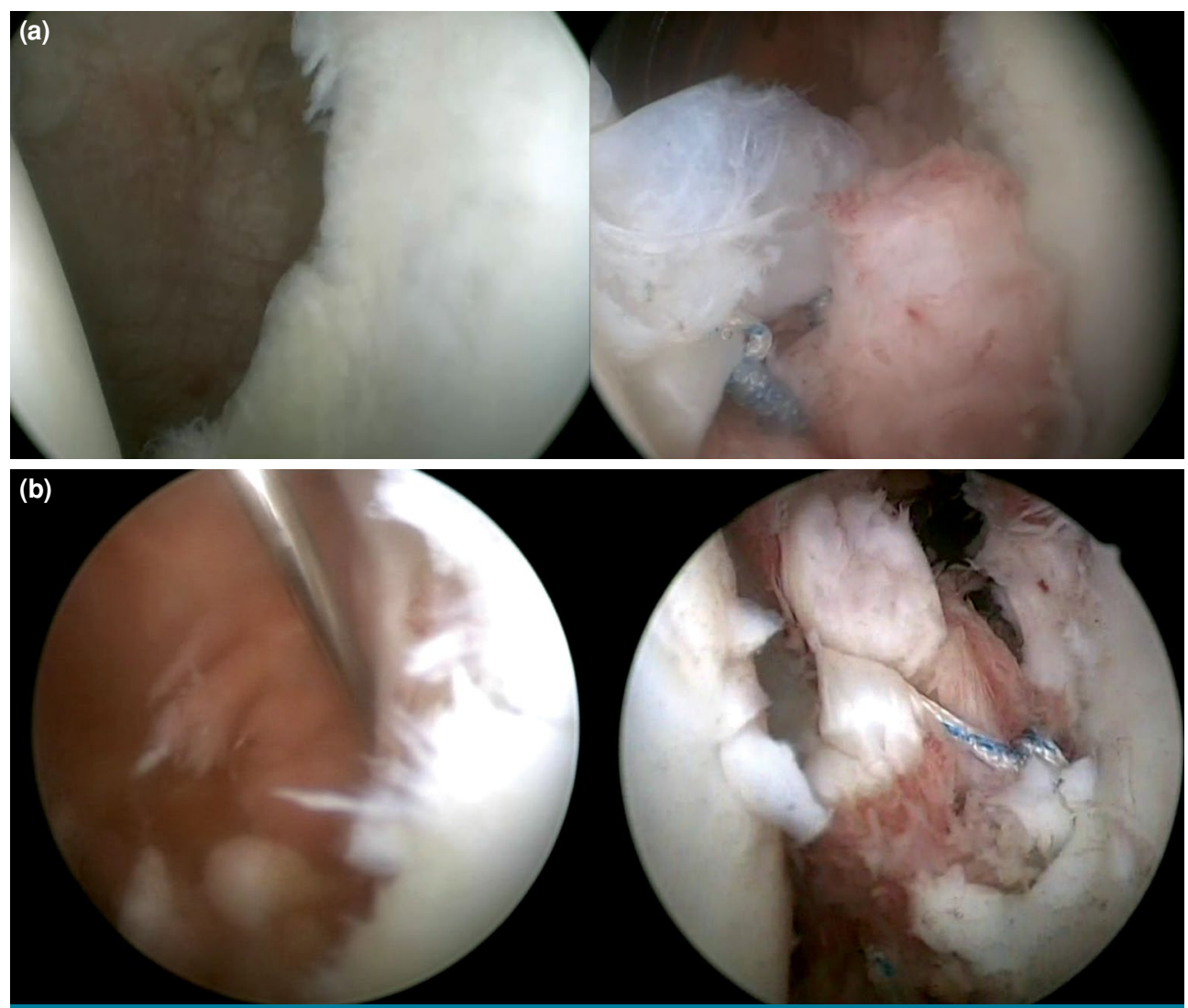

FIGURE 2. (a) Arthroscopic view of Bankart repair using two double-loaded suture anchors. (b) Arthroscopic view of Bankart repair using three double-loaded suture anchors. 


\begin{tabular}{|lcc|}
\multicolumn{3}{|c|}{ TABLE I } \\
Baseline demographic and clinical characteristics of patients \\
\hline & Group 1 & Group 2 \\
\hline Age (year) & 31.6 & 29.9 \\
Sex & & \\
$\quad$ Male & 16 & 22 \\
Female & 1 & 1 \\
Non-dominant hand & 3 & 2 \\
Joint laxity & 0 & 0 \\
Hill-Sacs lesion $(<5 \mathrm{~mm})$ & 7 & 8 \\
Glenoid fractures & 0 & 0 \\
\hline
\end{tabular}

\begin{tabular}{|lcc|}
\multicolumn{3}{c|}{$\begin{array}{c}\text { TABLE II } \\
\text { Forms of trauma }\end{array}$} \\
\hline Contact sports & Group 1 & Group 2 \\
\hline Traffic accident & 10 & 14 \\
Trauma & 1 & 2 \\
\hline
\end{tabular}

external rotation gain at 10 weeks. Active shoulder movements were started after eight weeks. Our goal was to provide the full ROM of the shoulder at three months. All patients were allowed to participate in non-contact sports three months after the operation. Heavy work and sports were allowed six months after the operation.

\section{Statistical analysis}

Statistical analysis was performed using the IBM SPSS version 22.0 software (IBM Corp., Armonk, NY, USA). Descriptive data were expressed in mean \pm standard deviation (SD), median (min-max) or number and frequency. The scores of both groups were assessed statistically by a paired independent t-test. A $p$ value of $<0.05$ was considered statistically significant.

\section{RESULTS}

Although 53 patients who underwent labral Bankart repair were screened initially, at the end of the follow-up, 13 patients were unable to be reached. Therefore, 40 patients with complete follow-up data were included in the analysis. No superficial or deep infection, or intra- and postoperative neurovascular complications were observed.

The mean follow-up was $48.9 \pm 18.6$ (range, 24 to 86 ) months in Group 1 and 51.8 \pm 22.2 (range, 24 to 86) months in Group 2. In Group 1, three patients had anterior glenohumeral instability on the non-dominant side, while two patients had on the non-dominant side in Group 2. Joint laxity was not present in both groups (Table I). The HillSachs lesion was below $5 \mathrm{~mm}$ in seven patients in Group 1 and in eight patients in Group 2.

In Group 1, anterior glenohumeral instability occurred in 10 patients with contact sports, in one patient by traffic accident, and in six patients after trauma. In Group 2, anterior glenohumeral instability occurred in 14 patients with contact sports, in two patients by traffic accident, and in seven patients after trauma (Table II). In the arthroscopic evaluation of all patients in terms of glenoid and humeral defects, the lesion was on-tract. Anterior labroligamentous periosteal sleeve avulsion lesions were present in four patients in Group 1 and five patients in Group 2. No humeral avulsion of the glenohumeral ligament lesions were observed in either group. None of the patients had glenoid fractures in either group. The mean time from the first dislocation to surgery was $70 \pm 74.1$ (range, 3 to 180) months in Group 1 and $67.8 \pm 72.9$ (range, 3 to 240 ) months in Group 2. In Group 1, the mean number of dislocations before surgery was $6.5 \pm 7.3$ (range, 1 to 18), while it was $6.2 \pm 7.7$ (range, 2 to 15) in Group 2. There was no significant difference in the mean time from the first dislocation to surgery $(p>0.001)$ and number of dislocations before surgery $(p>0.001)$ between the two groups (Table III).

\begin{tabular}{|c|c|c|c|c|c|}
\hline \multicolumn{6}{|c|}{$\begin{array}{c}\text { TABLE III } \\
\text { Follow-up and timing of surgery }\end{array}$} \\
\hline & \multicolumn{2}{|c|}{ Group 1} & \multicolumn{2}{|c|}{ Group 2} & \multirow[b]{2}{*}{$p$} \\
\hline & $\mathrm{n}$ & Mean \pm SD & $n$ & Mean \pm SD & \\
\hline Follow-up period & & $48.9 \pm 18.6$ & & $51.8 \pm 22.2$ & 0.667 \\
\hline Time between first dislocation with surgery & & $70 \pm 74.1$ & & $67.8 \pm 72.9$ & 0.925 \\
\hline Preoperative dislocation number & 6.5 & & 6.2 & & 0.712 \\
\hline
\end{tabular}




\begin{tabular}{|c|c|c|c|}
\hline \multicolumn{4}{|c|}{$\begin{array}{r}\text { TABLE IV } \\
\text { CSS and RS scores, and external ro }\end{array}$} \\
\hline & Group 1 & Group 2 & \\
\hline & Mean $\pm S D$ & Mean $\pm S D$ & $p$ \\
\hline CSS & $94.2 \pm 7.8$ & $95.4 \pm 4.1$ & 0.512 \\
\hline RS & $95.6 \pm 4.6$ & $96.3 \pm 3.8$ & 0.593 \\
\hline External rotation lose & $1.9 \pm 2.7$ & $2.2 \pm 2.4$ & 0.719 \\
\hline
\end{tabular}

The mean return to heavy work and sports was 6 months. In Group 1, the mean external rotation loss was $1.8 \pm 2.6^{\circ}$ (range, 0 to 7 ), while it was $2.1 \pm 2.4^{\circ}$ (range, 0 to 5) in Group 2. The two groups were also compared with RS and CSS scores at the at the end of the follow-up. There was no significant difference in CSS ( $p>0.001)$ and RS ( $p>0.001)$ scores between the two groups (Table IV).

Three of our patients had recurrent dislocation. Two patients in Group 1 and one patient in Group 2 experienced recurrent dislocation. Recurrent dislocations occurred after major trauma. In Group 1 , shoulder recurrence occurred in the first patient due to a fall while playing soccer and due to a fall while skiing in the second patient. In Group 2, recurrence occurred due to fall while playing soccer. No recurrence was observed during daily activities in any of the groups. At the end of the follow-up, there was also complete shoulder ROM in both groups. There was no significant difference between the groups in terms of recurrence and shoulder movement limitation, particularly external rotation.

\section{DISCUSSION}

In this study, we compared the clinical results between two double-loaded suture anchors and three double-loaded suture anchors in arthroscopic Bankart repair. Our study results showed no significant difference in terms of clinical results between the groups.

The number of suture anchors used in arthroscopic Bankart repair is still controversial. There is no consensus on the use of suture anchor numbers in the literature. Many authors recommend the use of three or four suture anchors to reduce recurrent instability in Bankart repair, and this recommendation is largely based on a retrospective review of single-loaded suture anchor use. ${ }^{[10]}$ However, there are also some studies reporting that the use of fewer suture anchors in Bankart repair compromises the stability of the shoulder and may increase the risk of recurrence. ${ }^{[3,5,11-14]}$
In biomechanical studies, it has been reported that the use of more than three suture anchors has no effect on clinical outcomes and does not make a difference in terms of stability. ${ }^{[15,16]}$ In recent years, different techniques and suture types have been used to reduce the number of suture anchors in arthroscopic Bankart repair. ${ }^{[8,17-23]}$ Successful results have been reported in arthroscopic Bankart repair using purse string technique using a single knotless suture. ${ }^{[16,17]}$ Similarly, in comparative clinical and biomechanical studies performing Bankart repair using double-loaded suture anchors, successful results were obtained by using less suture anchors. ${ }^{[10,16-19]}$ Theoretically, in Bankart repair with double-loaded suture anchors, each anchor can capture a larger portion of the capsulolabral complex and provide sufficient capsular plication to reduce joint volume. In arthroscopic Bankart repair, narrowing the capsule volume, as well as repairing the capsulolabral junction with good fixation, is effective in increasing clinical success. There are clinically different applications of capsular plication. Some surgeons have emphasized that only anterior plication may be sufficient. ${ }^{[2,25]}$ However, some others have advocated that capsular plication performed with the purse string technique would provide sufficient stability. ${ }^{[7,16]}$ In our study, we used a technique in which we reduced the capsule volume by providing capsular plication from inferior to superior, providing both medial and superior shift. We believe that double-loaded suture anchors render a stronger fixation of the capsule, compared to single-loaded suture anchors. Thus, we attempt to minimize the complications related to the use of multiple suture anchors. A glenoid rim fracture has been described as case report in the literature. Banerjee et al. ${ }^{[26]}$ reported a glenoid rim fracture in three patients in Bankart repair using three and four suture anchors. Therefore, we hypothesized that we could reduce the complications, such as cost and risk of fracture in the glenoid rim, by using a lesser number of double-loaded suture anchors.

In a successful arthroscopic Bankart repair, the repair of the dissociated and enlarged capsulolabral complex to the glenoid anterior increases the clinical success rate. In recent years, the number of suture types in different materials and configurations used for this purpose is quite high. However, there is no gold standard in the literature regarding the use of suture anchor numbers. Currently, the use of knotless suture anchors has increasingly become widespread. Even in clinically successful results, some biomechanical studies have shown that they are less resistant than knot-tying suture anchors. ${ }^{[27]}$ 
Similar biomechanical studies were conducted with single-loaded and double-loaded anchors. ${ }^{[10]}$ Aydin et $a l^{\left[{ }^{[8]}\right.}$ found that recurrence was higher in the group in which two knotless suture anchors were used. In this study, the authors found no significant difference between the two groups in terms of functional outcomes. In another study, Natera et al. ${ }^{[7]}$ reported good results in 94\% and recurrence in 5.4\% of the patients undergoing arthroscopic Bankart repair using a single absorbable suture anchor. However, in our study, there was no significant difference in the recurrence rate between the groups. ${ }^{[28]}$ Although it has advantages such as the absence of a knot in the joint, no damage to the articular surface or shorter operation time, Nho et al. ${ }^{[9]}$ showed, in their biomechanical cadaver study, that the knotless suture anchors were more durable than the knot-tying ones in recurrent loading. They also reported that there was no biomechanical difference between the single-loaded and doubleloaded suture anchors in this study. Godinho and et al. ${ }^{[17]}$ found no statistically significant difference in the rate of dislocation recurrence and clinical outcomes after arthroscopic Bankart repair using three or more single-loaded and double-loaded anchors. Kamath et al. ${ }^{[10]}$ showed that the use of two double-loaded anchors in biomechanical cadaver studies was superior to three single-loaded anchors biomechanically.

Many biomechanical and clinical studies have reported loss of external rotation after capsular plication. ${ }^{[27,29,30]}$ Godinho et al. ${ }^{[17]}$ found that the use of three or more double-loaded suture anchors, in particular, limited the use of external rotation in adduction and abduction. However, the authors reported that external rotation in adduction loss had no effect on recurrence rates and functional scores. In these studies, we believe that the limitation of $\mathrm{ROM}$ is due to excessive stretching of the anterior capsule. However, some studies have also reported that capsular plication does not lead to loss of external rotation. ${ }^{[8,31,32]}$ In our study, there was no significant difference between the two groups after capsular plication and no significant external rotation loss was observed in any of the patients. ${ }^{[28]}$

One of the main limitations of the present study is that the number of cases is relatively small. Another limitation is its retrospective design. However, the main strength of this study is that, to the best of our knowledge, there is no study comparing the result of two double-loaded and three double-loaded suture anchors used in arthroscopic Bankart repair.
In conclusion, our study results suggest that stability is not correlated with the use of either two versus three double-loaded suture anchors in arthroscopic Bankart repairs. In addition, recurrence rate and functional results are comparable with the use of both sutures. Further large-scale, prospective studies are needed to confirm these findings.

\section{Declaration of conflicting interests}

The authors declared no conflicts of interest with respect to the authorship and/or publication of this article.

\section{Funding}

The authors received no financial support for the research and/or authorship of this article.

\section{REFERENCES}

1. Sarıkaya B, Bozkurt C, Gül O, Bekin Sarıkaya PZ, Sipahioğlu S, Altay MA. Comparison of the clinical results of isolated Bankart and SLAP 5 lesions after arthroscopic repair. Jt Dis Relat Surg 2020;31:223-9.

2. Hobby J, Griffin D, Dunbar M, Boileau P. Is arthroscopic surgery for stabilisation of chronic shoulder instability as effective as open surgery? A systematic review and meta-analysis of 62 studies including 3044 arthroscopic operations. J Bone Joint Surg [Br] 2007;89:1188-96.

3. Boileau P, Villalba M, Héry JY, Balg F, Ahrens P, Neyton L. Risk factors for recurrence of shoulder instability after arthroscopic Bankart repair. J Bone Joint Surg [Am] 2006;88:1755-63.

4. van der Linde JA, van Kampen DA, Terwee CB, Dijksman LM, Kleinjan G, Willems WJ. Long-term results after arthroscopic shoulder stabilization using suture anchors: an 8- to 10-year follow-up. Am J Sports Med 2011;39:2396-403.

5. Kim SH, Ha KI, Kim SH. Bankart repair in traumatic anterior shoulder instability: open versus arthroscopic technique. Arthroscopy 2002;18:755-63.

6. Levy O, Matthews T, Even T. The "purse-string" technique: an arthroscopic technique for stabilization of anteroinferior instability of the shoulder with early and medium-term results. Arthroscopy 2007;23:57-64.

7. Natera LG, Consigliere P, Witney-Lagen C, Bruguera J, Sforza G, Atoun E, et al. The "Purse String" procedure for recurrent anterior glenohumeral instability: a simple technique to achieve bankart repair, capsular shift, and a good labral bumper. Arthroscopy Techniques 2017;6:e1245-e1251.

8. Aydin N, Karaismailoglu B, Harbiyeli E, Ozsahin MK. Can capsular plication compensate the lack of one suture anchor in an arthroscopic three suture anchor Bankart repair? A comparative study. Acta Orthop Traumatol Turc 2019;53:266-71.

9. Nho SJ, Frank RM, Van Thiel GS, Wang FC, Wang VM, Provencher MT, et al. A biomechanical analysis of anterior Bankart repair using suture anchors. Am J Sports Med 2010;38:1405-12.

10. Kamath GV, Hoover S, Creighton RA, Weinhold P, Barrow A, Spang JT. Biomechanical analysis of a double-loaded glenoid anchor configuration: can fewer anchors provide equivalent fixation? Am J Sports Med 2013;41:163-8. 
11. Parmar RS, Kapoor S, Sharma B. Arthroscopic Bankart repair following traumatic recurrent shoulder dislocation: A prospective review of 30 cases. J Orthop Surg (Hong Kong) 2019;27:2309499019832708.

12. Sedeek SM, Tey IK, Tan AH. Arthroscopic Bankart repair for traumatic anterior shoulder instability with the use of suture anchors. Singapore Med J 2008;49:676-81.

13. Walch G, Boileau P, Levigne C, Mandrino A, Neyret P, Donell S. Arthroscopic stabilization for recurrent anterior shoulder dislocation: results of 59 cases. Arthroscopy 1995;11:173-9.

14. Randelli P, Ragone V, Carminati S, Cabitza P. Risk factors for recurrence after Bankart repair a systematic review. Knee Surg Sports Traumatol Arthrosc 2012;20:2129-38.

15. Martetschläger F, Michalski MP, Jansson KS, Wijdicks CA, Millett PJ. Biomechanical evaluation of knotless anterior and posterior Bankart repairs. Knee Surg Sports Traumatol Arthrosc 2014;22:2228-36.

16. Witney-Lagen C, Perera N, Rubin S, Venkateswaran B. Fewer anchors achieves successful arthroscopic shoulder stabilization surgery: 114 patients with 4 years of follow-up. J Shoulder Elbow Surg 2014;23:382-7.

17. Godinho GG, Freitas JM, França Fde O, de Lago E Santos FM, Aragão AA, Barros MK. Bankart arthroscopic procedure: comparative study on use of double or singlethread anchors after a 2-year follow-up. Rev Bras Ortop 2014;50:94-9.

18. Kim KC, Shin HD, Cha SM, Kim JH. Arthroscopic double-loaded single-row repair in chronic traumatic anterior shoulder dislocation. Arch Orthop Trauma Surg 2012;132:1515-20.

19. Chen JS, Novikov D, Kaplan DJ, Meislin RJ. Effect of additional sutures per suture anchor in arthroscopic bankart repair: A review of single-loaded versus doubleloaded suture anchors. Arthroscopy 2016;32:1415-20.

20. Cho NS, Lubis AM, Ha JH, Rhee YG. Clinical results of arthroscopic bankart repair with knot-tying and knotless suture anchors. Arthroscopy 2006;22:1276-82.

21. Brown BS, Cooper AD, McIff TE, Key VH, Toby EB. Initial fixation and cyclic loading stability of knotless suture anchors for rotator cuff repair. J Shoulder Elbow Surg 2008;17:313-8.

22. Hayashida K, Yoneda M, Mizuno N, Fukushima S,
Nakagawa S. Arthroscopic Bankart repair with knotless suture anchor for traumatic anterior shoulder instability: results of short-term follow-up. Arthroscopy 2006;22:620-6.

23. Garofalo R, Mocci A, Moretti B, Callari E, Di Giacomo G, Theumann $\mathrm{N}$, et al. Arthroscopic treatment of anterior shoulder instability using knotless suture anchors. Arthroscopy 2005;21:1283-9.

24. Mayer SW, Kraszewski AP, Skelton A, Kontaxis A, Warren $\mathrm{R}$. What are the effects of capsular plication on translational laxity of the glenohumeral joint: A study in cadaveric shoulders. Clin Orthop Relat Res 2018;476:1526-36.

25. Sodl JF, McGarry MH, Campbell ST, Tibone JE, Lee TQ. Biomechanical effects of anterior capsular plication and rotator interval closure in simulated anterior shoulder instability. Knee Surg Sports Traumatol Arthrosc 2016;24:365-73.

26. Banerjee $S$, Weiser L, Connell D, Wallace AL. Glenoid rim fracture in contact athletes with absorbable suture anchor reconstruction. Arthroscopy 2009;25:560-2.

27. Bohnsack $M$, Bartels $B$, Ostermeier $S$, Rühmann $O$, Wellmann M, Mansouri F, et al. Biomechanical stability of an arthroscopic anterior capsular shift and suture anchor repair in anterior shoulder instability: a human cadaveric shoulder model. Knee Surg Sports Traumatol Arthrosc 2009;17:1493-9.

28. Atik OŞ. Is there something new and interesting in my article? Eklem Hastalik Cerrahisi 2019;30:69.

29. Kim DW, Kim CK, Jung SW. An arthroscopic pleated capsular shift for recurrent anterior dislocation of the shoulder. Knee Surg Sports Traumatol Arthrosc 2012;20:2579-84.

30. Castagna A, Borroni M, Delle Rose G, Markopoulos N, Conti M, Vinci E, et al. Effects of posterior-inferior capsular plications in range of motion in arthroscopic anterior Bankart repair: a prospective randomized clinical study. Knee Surg Sports Traumatol Arthrosc 2009;17:188-94.

31. Park JY, Chung SW, Kumar G, Oh KS, Choi JH, Lee D, et al. Factors affecting capsular volume changes and association with outcomes after Bankart repair and capsular shift. Am J Sports Med 2015;43:428-38.

32. Levy DM, Gvozdyev BV, Schulz BM, Boselli KJ, Ahmad CS. Arthroscopic anterior shoulder stabilization with percutaneous assistance and posteroinferior capsular plication. Am J Orthop (Belle Mead NJ) 2014;43:364-9. 